\title{
On the history of pion-pion scattering
}

\author{
Juerg Gasser ${ }^{* \dagger}$ \\ Albert Einstein Center for Fundamental Physics \\ Institute for Theoretical Physics \\ University of Bern \\ Sidlerstrasse 5, 3012 Bern, Switzerland \\ E-mail: gasseraitp.unibe.ch
}

Highlights in the history of pion-pion scattering are discussed, starting with Yukawa's hypothesis that pion exchange is responsible for nuclear forces, and ending with the NA48/2 analysis of $K_{e} 4$ and $K \rightarrow 3 \pi$ decays. No claim for completeness is made.

International Workshop on Effective Field Theories: from the pion to the upsilon 2-6 February 2009

Valencia, Spain

${ }^{*}$ Speaker.

$\dagger$ The Albert Einstein Center for Fundamental Physics is supported by the "Innovations- und Kooperationsprojekt C-13" of the "Schweizerische Universitätskonferenz SUK/CRUS". This work was in addition supported by the Swiss National Science Foundation, by EU MRTN-CT-2006-035482 (FLAVIAnet) and by the Helmholtz Association through funds provided to the virtual institute "Spin and strong QCD" (VH-VI-231). 


\section{Introduction}

About 75 years have passed since Yukawa's hypothesis that pions are the source of nuclear forces [1], about 60 years since their experimental discovery [2], and 50 years since first attempts to measure the strength of their interactions [3]. The progress made since these days can be illustrated by going back to the 1960 Rochester conference [4]. There one finds the contribution from Batusov et al. [5], who discuss the determination of the charge-exchange cross section $\sigma_{\pi^{+} \pi^{-} \rightarrow \pi^{0} \pi^{0}}$. In their article, the authors tabulate "... all the data available at present concerning the amplitudes of $S$-waves $\pi \pi$ scattering, see Table 1 . It is seen that the size of the scattering lengths was largely unknown, even their sign was not yet determined. To some authors, it was even not certain that $\pi \pi$ interactions do exist. Indeed, in Ref. [6], one can read the statement: "There are some phenomena which might be considered to show the existence of pion pion interactions." Today, the low-energy $\pi \pi$ amplitude is known to very high precision, in the percent range, both, theoretically and experimentally. On the theory side, this has become possible through the use of Roy equations and of chiral perturbation theory (ChPT), whereas on the experimental side, the progress was on the one hand due to the incredible increase of statistics. On the other hand, new experimental technique have become available, that allowed one to pin down the $\pi \pi$ amplitude to very high precision. Here, a very nice interplay between theory and experiment has emerged, which has lead to an improved knowledge of the $\pi \pi$ interactions.

\begin{tabular}{r|c|c|c|l} 
Reaction & $a_{0}$ & $a_{2}$ & $a_{2}-a_{0}$ & References \\
\hline$K \rightarrow 3 \pi$ & & $\sim 1$ & & {$[7]$} \\
$\pi N \rightarrow \pi N$ & $\sim 1$ & & & {$[6]$} \\
$K \rightarrow 3 \pi$ & -1 & -0.3 & 0.7 & {$[8]$} \\
$K \rightarrow 3 \pi$ & -0.8 & -0.48 & 0.3 & {$[9]$} \\
$\pi N \rightarrow \pi N$ & $\sim 1$ & & & {$[10]$} \\
$\pi^{-} p \rightarrow \pi^{+} \pi^{-} n$ & & & $-(0.35 \pm 0.30)$ & {$[5]$}
\end{tabular}

Table 1: Knowledge of $\pi \pi \mathrm{S}$-wave scattering lengths at the time of the Rochester Conference 1960, in chronological order (according to the submission of the articles). The result of Ref. [7] concerns the absolute value of $a_{2}$. The table is set up according to the information provided in Ref. [4].

It impossible to provide here a complete history of these very interesting developments. It is not only the lack of space which prevents me to do this, but also my ignorance of early developments. For this reason, I shall simply mention several highlights ${ }^{1}$ - quite naturally, the choice will be a subjective one, and I apologize to all who feel that I should have mentioned their contribution to the topic.

\footnotetext{
${ }^{1}$ For the survey before 1965, I profited from the work of Six and Artru [11], and of Brown and Rechenberg [12].
} 


\section{Prediction and discovery of pions}

In the year 1934, Yukawa submitted an article where he proposed that the exchange of (spin zero) particles of mass about 200 times the electron mass and charge $\pm e$ are responsible for the nuclear forces [1]. As the mass needed was between the one of the electron and of the proton, the particles were later called mesotrons [13], then mesons [2] for short. They were discovered thirteen years later by Lattes, Muirhead, Occhialini and Powell [2], who examined plates exposed in the Bolivian Andes at a height of 5500 meters. There were two different kind of mesons of slightly different masses, a fact which first led to some confusion. As could be read off from the tracks on the plates, the heavier mesons disintegrated into secondary ones. The authors represented the primary mesons by the symbol $\pi$, and the secondary ones by $\mu$ [the symbol $\mu$ was already introduced in Ref. [14]] - pions and muons. The prediction and discovery were rewarded with Nobel Prizes: Yukawa in 1949, Powell in 1950. Negative (positive) pions were first artificially produced in the 184-inch Berkeley cyclotron [15] ([16]) in 1948. In the same year, Wigner mentions the discontinuity of the energy derivative of scattering cross sections at the threshold of a new channel [17]. We will come back to this observation below.

\section{1960 - 1970: Interacting pions}

First methods for extracting $\pi \pi$ cross sections from experimental data were already developed in the late fifties [18], and sigma models, both, linear and non linear ones, were investigated as Quantum Field Theories (QFT) of $\pi \pi$ interactions [19]. Goldberger and Treiman wrote their famous paper [20] on a relation between weak and strong interaction coupling constants, $g_{\pi N N} F_{\pi}=g_{A} m_{N}$. Nambu [21], in an attempt to provide more insight into the reason for this relation, pointed out that the mass of pions is small, because strong interactions have an approximate chiral symmetry which is spontaneously broken. He was awarded the Nobel prize for his observation in the year 2008. In this framework, the Goldberger-Treiman relation emerges very naturally, see also the article by Gell-Mann and Lévy [19], whose abstract starts with the sentence "In order to derive in a convincing manner the formula of Goldberger and Treiman,...”. In Ref. [22], Treiman wrote an interesting article about the background of the derivation of that relation - a derivation which was not well accepted in the literature. He comments the criticisms: "...but frankly, I would have preferred being treated with a bit more respect".

Also in the late fifties, Wigner's observation [17] was developed in more details by many authors - see e.g. the article by Newton [23] and by Fonda and Newton [24] for a short review. Based on the work of Refs. [24, 25], Budini and Fonda [26] investigated the threshold singularities in $K \rightarrow \pi^{+} \pi^{0} \pi^{0}$ decays in detail and showed that the partial decay rate $d \Gamma / d_{E_{\pi}^{+}}$will generate a cusp, whose strength is given by the charge exchange amplitude for the reaction $\pi^{+} \pi^{-} \rightarrow \pi^{0} \pi^{0}$. They provided an analytic formula for the cusp and pointed out that, in principle, this decay allows one to measure a particular combination of scattering lengths $a_{0}-a_{2}$. I find it quite amazing that this method was described only 14 years after the discovery of the pion. It is amazing even more when observing that the method only works because the pion masses are split. [The difference $M_{\pi}-M_{\pi^{0}}$ was measured in Ref. [27] to be $10.6 \pm 2.0$ electron masses. ${ }^{2}$ ]

\footnotetext{
${ }^{2}$ The symbols $M_{\pi}\left(M_{\pi^{0}}\right)$ denote the charged (neutral) pion mass throughout.
} 
There were only a handful of $K \rightarrow 3 \pi$ decays available in those days, and it was thus impossible for Budini and Fonda to determine the $\pi \pi$ scattering lengths. The method was then forgotten, and rediscovered 45 years later by Cabibbo [28], while analysing data from these decays, as measured copiously by the NA48/2 collaboration [29]. I will come back to this point below.

Still in this period belong the observations that

- $\pi^{+} \pi^{-}$bound states exist (pionium) [30]. The ground state decays dominantly into two neutral pions, and the width for this decay is of the form

$$
\Gamma_{\pi^{0} \pi^{0}}=C\left(a_{0}-a_{2}\right)^{2} .
$$

In other words, by measuring the lifetime for this decay, one can again measure (the absolute value of) the combination $a_{0}-a_{2}$.

- Final state interactions in $K^{+} \rightarrow \pi^{+} \pi^{-} e^{+} v_{e}$ allow one to measure the phase difference $\delta_{0}^{0}-$ $\delta_{1}^{1}$ [31]. [The first $K^{+} \rightarrow \pi^{+} \pi^{-} e^{+} v$ decay was reported in Ref. [32], with a camera lucida drawing of the event.]

- Current algebra leads to the relation

$$
a_{0}=\frac{7 M_{\pi}^{2}}{32 \pi F_{\pi}^{2}}=0.159 \quad[33] .
$$

Here, $F_{\pi}$ denotes the pion decay constant $F_{\pi}=92.4 \mathrm{MeV}$. Weinberg [33] notes that this result is very much smaller than every one else had thought. In his 1997 article [34], he says that the result Eq. (3.2) was the greatest defeat of S-matrix theory [which predicted large scattering lengths].

\section{1970 - 1990: Foundation of theory and experiment}

In this period fall many important events.

- Roy did set up integral equations for partial waves in elastic $\pi \pi$ scattering [35]. Due to the large symmetry of these reactions (there is only one Lorentz invariant amplitude in the case of an isospin symmetric world), the absorptive parts in the pertinent dispersion relations are given by the imaginary part of partial wave amplitudes evaluated in the physical region. Moreover, invoking unitarity, these dispersion relations turn into an infinite set of integral equations for phase shifts. For a review of early numerical work on these equations, I refer the reader to the article by Morgan and Pennington [36]. Froggatt and Peterson [37] used these methods to analyse the Geneva-Saclay measurements of $K_{e 4}$ decays performed at the CERN PS in 1977 [38], see below.

- Lehmann [39] pointed out that chiral symmetry, analyticity and unitarity pin down the elastic $\pi \pi$ amplitude in a well defined expansion, known as ChPT in our days, at next to leading order, up to two unknown constants (in the chiral limit). Also in these years, Ecker and Honerkamp used covariant chiral perturbation theory and superpropagators to determine the $\pi \pi$ amplitude at the same order [40]. 
- In 1973, Quantum Chromo Dynamics (QCD) was proposed as the theory of strong interactions [41].

- In 1977, the first high-statistic experiment on $K_{e 4}$ was performed at CERN [38] [30 000 events]. Based on the analysis of the data and on their solutions of the Roy equations, Froggatt and Petersen came up with

$$
a_{0}=0.26 \pm 0.05 \quad[37]
$$

The value $a_{0}=0.28 \pm 0.05$ quoted in Ref. [38] is based on slightly older solutions [42] of the Roy equations. The comment in Ref. [38] on the value $a_{0}=0.28 \pm 0.05$ sounds quite amusing in our days: The value for $a_{0}$ is somewhat above the original Weinberg prediction, but it appears that this prediction can be revised without any fundamental change in current algebra. Today, we know that this is not correct: Had it turned out that the scattering length indeed is of this size, fundamental properties of QCD would be very different from what we now know [43, 44]. I come back to this point below.

There is a second amusing remark concerning the above result Eq. (4.1). While analysing the data, isospin breaking corrections, induced by real and virtual photons, were taken into account to purify the data from these effects. We now know that this analysis did not take into account one important effect, generated by the mass difference of the charged and neutral pion $[45,46]$. Once this effect is taken care of, the value of the scattering length in the Geneva-Saclay experiment Eq. (4.1) becomes $a_{0}=0.23 \pm 0.05$ [47] - a very substantial correction. If this had been known before, many of the discussions on the nonstandard scenario of chiral symmetry breaking would presumably never have taken place.

After 1977, interest in Roy equations waned.

- The low energy effective theory of QCD (ChPT) was set up by Weinberg in 1979 [48], and, in a systematic framework, in Refs. [49, 50] in 1984 and 1985. ChPT was used to calculate the $\pi \pi$ scattering amplitude to one loop, with the result

$$
a_{0}=0.20 \pm 0.01 \quad[49]
$$

The uncertainty does not include effects from still higher order corrections.

- In 1986, Lüscher pointed out that scattering lengths in massive QFT can be measured by investigating volume effects in the energy levels of particles confined to a box [51]. This in particular opened the way for the evaluation of $\pi \pi$ scattering lengths in the framework of lattice QCD.

\section{1990 - 2005: High precision at low energies}

In the fifteen years from 1990 - 2005, knowledge of the elastic $\pi \pi$ scattering amplitude increased dramatically. 
- Nearly twenty years ago, in 1991, the concept of generalized ChPT [GChPT] was set up [43, 44]. The main observation was that the chiral condensate $\langle 0|\bar{q} q| 0\rangle$ may be small or even vanishing - there was no compelling experimental observation against this scenario. One of the main reasons for these investigations was the fact that a large scattering length $a_{0}$ is allowed in this framework. I witnessed many heated debates about this framework over the years. As far as I can judge, the final blow to this scenario was the observation that the NA48/2 results, after isospin breaking corrections applied, are in perfect agreement with the standard scenario of spontaneously chiral symmetry breaking, and therefore with a small scattering length.

- The DIRAC proposal [52] to determine $\left|a_{0}-a_{2}\right|$ via the lifetime measurement of pionium was approved by the research board of CERN at a meeting on February 26, $1996^{3}$. The DIRAC collaboration has provided meanwhile [53] first results for $\left|a_{0}-a_{2}\right|$, see below.

- An $S$-matrix calculation of the elastic $\pi \pi$ amplitude at order $p^{6}$ was published in 1996 [44]. As the authors did not perform a standard two-loop calculation in the framework of QFT, they missed some information which is reachable with the latter method, used in Ref. [54] to evaluate the same amplitude at the same accuracy, with all non analytic terms included.

- Meißner, Müller and Steininger pointed out in 1997 [55] that the pion mass difference generates a cusp in the reaction $\pi^{0} \pi^{0} \rightarrow \pi^{0} \pi^{0}$. The strength of the cusp is proportional to $a_{0}-a_{2}$.

- Driven by the fact that the $\pi \pi$ amplitude was available to two loops, there was a revival of the use of Roy equations $[56,57]$ to analyse $\pi \pi$ scattering. The main observation was the fact that the scattering amplitude can be calculated quite precisely, once the scattering lengths $a_{0}, a_{2}$ are known, together with data above a center-of-mass energy of about 800 $\mathrm{MeV}$. Combining Roy equations and ChPT lead to a very sharp prediction of the isospin $I=0,2$ S-wave scattering lengths,

$$
a_{0}=0.220 \pm 0.005, a_{0}-a_{2}=0.265 \pm 0.004 \quad[58] .
$$

More threshold parameters are provided in the same reference. These values confirmed the results of Amoros et al. [59] (obtained in pure ChPT), although with smaller uncertainties. For comments on other determinations of the scattering lengths $a_{0,2}$ available in the literature, I refer the reader to Colangelo's contribution at KAON07 [60].

- In the years 2001-2003, the E865 collaboration in Brookhaven [61] analyzed more than $4 \times 10^{5} K_{e 4}$ decays, leading to

$$
a_{0}=0.216 \pm 0.013_{\text {stat }} \pm 0.002_{\text {syst }} \pm 0.002_{\text {theor }} \quad[61] .
$$

The apparently confirmed the small scattering length scenario, eliminating GChPT - see, however, below!

- Nemenov et al. proposed in 2002 [62] to use subtle quantum mechanical effects to measure $a_{0,2}$ to percent precision by investigating excited energy levels in pionium.

\footnotetext{
${ }^{3}$ I thank Leonid Nemenov for providing me with this information.
} 
- In an attempt to see effects of pionium production in $K^{+} \rightarrow \pi^{+} \pi^{0} \pi^{0}$, the NA48/2 collaboration observed a cusp-like structure in the differential decay distribution $d \Gamma / d_{E_{\pi^{+}}}$. Cabibbo [28] pointed out that the cusp is due to a subtle isospin breaking effect in the elastic $\pi \pi$ scattering amplitude, allowing one to measure $a_{0}-a_{2}$. As already mentioned, this is the effect discussed by Budini and Fonda in Ref. [26] nearly 50 years ago. The number of decays available now is overwhelming - the published analysis from 2005 [29] is based on $2.7 \times 10^{7}$ events! Using the representation of the amplitude as worked out in Ref. [63], the result is

$$
\begin{aligned}
a_{0}-a_{2} & =0.268 \pm 0.010_{\text {stat }} \pm 0.004_{\text {syst }} \pm 0.0013_{\text {ext }} \\
a_{2} & =-0.041 \pm 0.022_{\text {stat }} \pm 0.014_{\text {syst }} \quad[29]
\end{aligned}
$$

The fact that $K \rightarrow 3 \pi$ decays allow one to precisely determine $\mathrm{S}$-wave scattering lengths has lead to new theoretical developments [64, 65].

- The theory of hadronic atoms has also made important improvements in this period [66]. Using a non relativistic quantum field theory, the width of pionium for the decay into two neutral pions was found to be $[66,67]$

$$
\Gamma_{2 \pi^{0}}=\frac{2}{9} \alpha^{3} p^{*}\left(a_{0}-a_{2}\right)^{2}(1+\varepsilon) ; \varepsilon=(5.8 \pm 1.2) \times 10^{-2} .
$$

Here, $p^{*}$ is the (modulus of the) center-of-mass momentum of the outgoing neutral pions. Inserting the values Eq. (5.1) for $a_{0}-a_{2}$ gives [67]

$$
\tau_{2 \pi^{0}}=(2.9 \pm 0.1) \times 10^{-15} \mathrm{sec},
$$

while the experimental value is [53]

$$
\tau=\left(2.9_{-0.62}^{+0.49}\right) \times 10^{-15} \mathrm{sec},
$$

or

$$
\left|a_{0}-a_{2}\right|=0.264_{-0.020}^{+0.033} \quad[53]
$$

All in all, the experimental results $(5 \cdot 2,5.3,5.7)$ amount to three very nice confirmations of the predictions Eq. (5.1).

This was the situation around the year 2005 .

\section{2006 - 2009: A puzzle and its resolution}

In the year 2006, a surprise occurred: at the QCD conference in Montpellier (QCD06), Brigitte Bloch-Devaux reported [68] preliminary results of the analysis of a large amount of $K_{e 4}$ decays collected by the NA48/2 collaboration in 2003/2004. The numbers presented were

$$
a_{0}=\left\{\begin{array}{l}
0.256 \pm 0.011 \mathrm{NA} 48 / 2 \quad 370000 K_{e 4} \text { decays } \\
0.253 \pm 0.037 \text { Geneva-Saclay } \quad 30000 K_{e 4} \text { decays }
\end{array}[68] .\right.
$$




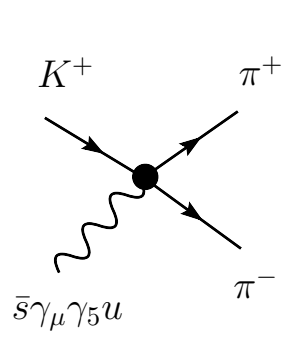

a)

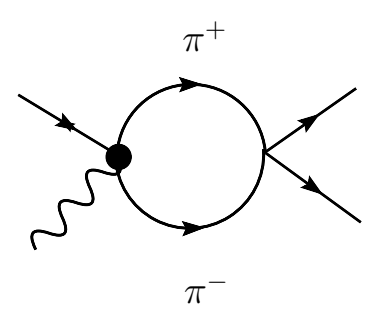

b)

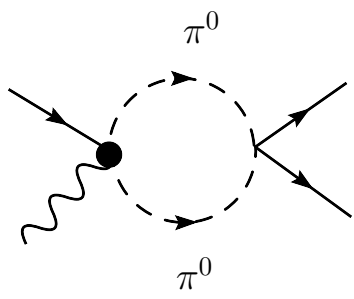

c)

Figure 1: Some of the graphs that contribute to the matrix element of the axial current at tree and one-loop order. The filled vertex indicates that the axial current also couples to a single kaon line. There are many additional graphs at one-loop order, not displayed in the figure.

Here, the first row refers to the NA48/2 data, where solutions to the Roy equations [56] had been used to analyse the data. The second row represents a fit to the old data [38], using the same Roy solutions, and thus confirms the result Eq. (4.1) which is based on old solutions to the Roy equations [37]. Several reports were given by members of the NA48/2 collaboration [69], underlining the large value for $a_{0}$ found in Ref. [68], see also Ref. [70].

So, because the statistics was increased by a factor of roughly 15 , there was practically no doubt that the scattering length $a_{0}$, as determined from $K_{e 4}$ decays, is larger than predicted by standard ChPT, and of the size found in 1977 [37] - a revival of the small condensate scenario?

The resolution of the puzzle came from an unexpected side. In spring 2007, we organized a two-days meeting at the University of Bern to discuss the issue with experimentalists and theorists. In the days after the meeting, the solution popped up: there is an isospin breaking effect that was not taken into account in the data analysis so far. It is substantial, goes into the right direction and has the correct size to put the NA48/2 data in perfect agreement with the chiral prediction [45].

The main observation was the following. In the NA48/2 experiment before summer 2007, the general purpose FORTRAN routine PHOTOS [71] was used to calculate electromagnetic corrections. In addition, the Sommerfeld factor was applied, to account for the Coulomb interaction between charged particles [72]. In these prescriptions to perform radiative corrections, one specific mechanism is not included. Namely, the kaon may decay first into a neutral pion pair, that then annihilates into two charged pions, or first decay into a charged pion pair, that then rescatters. In addition, a $\pi^{0} \eta$ mixing effect also occurs, see Figures 1 and 2. In the real world, the neutral pion mass is smaller than the charged one by about $4.6 \mathrm{MeV}$. As a result of this, these three contributions to the decay matrix element have not the same holomorphic structure: the neutral (charged) pion loop generates a branch point at $s_{\pi}=4 M_{\pi^{0}}^{2}$ (at $s=4 M_{\pi}^{2}$ ), and the phase of the relevant form factor is affected with a cusp, and does not vanish at the threshold $s=4 M_{\pi}^{2}$.

\subsection{Isospin breaking effects in $K_{e 4}$ decays}

In [45], the mentioned effects are investigated in several steps:

i) One assumes that the manner in which real and virtual photons are treated in the analysis of the NA48/2 experiment (PHOTOS + Sommerfeld factor) is a decent approximation to the 


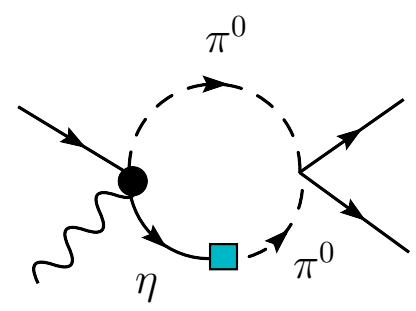

Figure 2: The contribution from $\pi^{0}-\eta$ mixing, at leading order in $m_{d}-m_{u}$. The filled square denotes the vertex from $\pi^{0}-\eta$ mixing. Otherwise, the notation is the same as in Figure 1.

effects generated by soft photons.

ii) This procedure misses the effects generated by the pion and kaon mass differences, and by the quark mass difference $m_{d}-m_{u}$. These must therefore be taken into account separately.

iii) ChPT is the appropriate tool to evaluate these contributions.

iv) One assumes that PHOTOS+Sommerfeld factor + mass effects provide a good approximation to the full isospin breaking contributions:

\section{Full isospin breaking effects $=$ Coulomb factor $\times$ PHOTOS $\times$ mass effects}

This allows one to also correct earlier analyses with the last factor. This should catch the main missing effects. According to iii), we therefore simply need to perform a ChPT calculation of the effects generated by the mass differences. The relevant diagrams at one-loop order are displayed in Figures 1,2. One finds that the phase becomes

$$
\delta_{0}^{0} \rightarrow \delta=\frac{1}{32 \pi F_{\pi}^{2}}\left\{\left(4 \Delta_{\pi}+s_{\pi}\right) \sigma+\left(s_{\pi}-M_{\pi^{0}}^{2}\right)\left(1+\frac{3}{2 R}\right) \sigma_{0}\right\}, s_{\pi} \geq 4 M_{\pi}^{2}
$$

with

$$
\Delta_{\pi}=M_{\pi}^{2}-M_{\pi^{0}}^{2}, \quad \sigma_{0}=\left(1-\frac{4 M_{\pi^{0}}^{2}}{s_{\pi}}\right)^{1 / 2}, \quad \sigma=\left(1-\frac{4 M_{\pi}^{2}}{s_{\pi}}\right)^{1 / 2}, \quad R=\frac{m_{s}-\hat{m}}{m_{d}-m_{u}},
$$

where $\hat{m}=\frac{1}{2}\left(m_{u}+m_{d}\right)$.The one-loop calculation performed in Refs. [73] is compatible with this expression, up to terms of order $\alpha_{\mathrm{QED}}\left(m_{d}-m_{u}\right)$. [Indeed, these references implicitly contain the effects considered here. Unfortunately, the manner in which the ChPT result is presented and analyzed cannot be used for the present purpose. In Ref. [46], the cusp in $K_{e 4}$ decays was investigated as well. The expressions presented there do not agree with Eq. (6.2), because these authors did not take into account derivative couplings of the $\pi \pi$ amplitude, as is dictated by chiral symmetry, and did not include the effect of the quark mass difference $m_{d}-m_{u}$.]

The result (6.2) is very interesting, for the following reasons. First, due to the presence of the phase space factor $\sigma_{0}$, the phase $\delta$ does not vanish at the threshold $s_{\pi}=4 M_{\pi}^{2}$. This unexpected behaviour of the phase is the cusp effect already experienced in $K \rightarrow 3 \pi$ decays, with the role of 


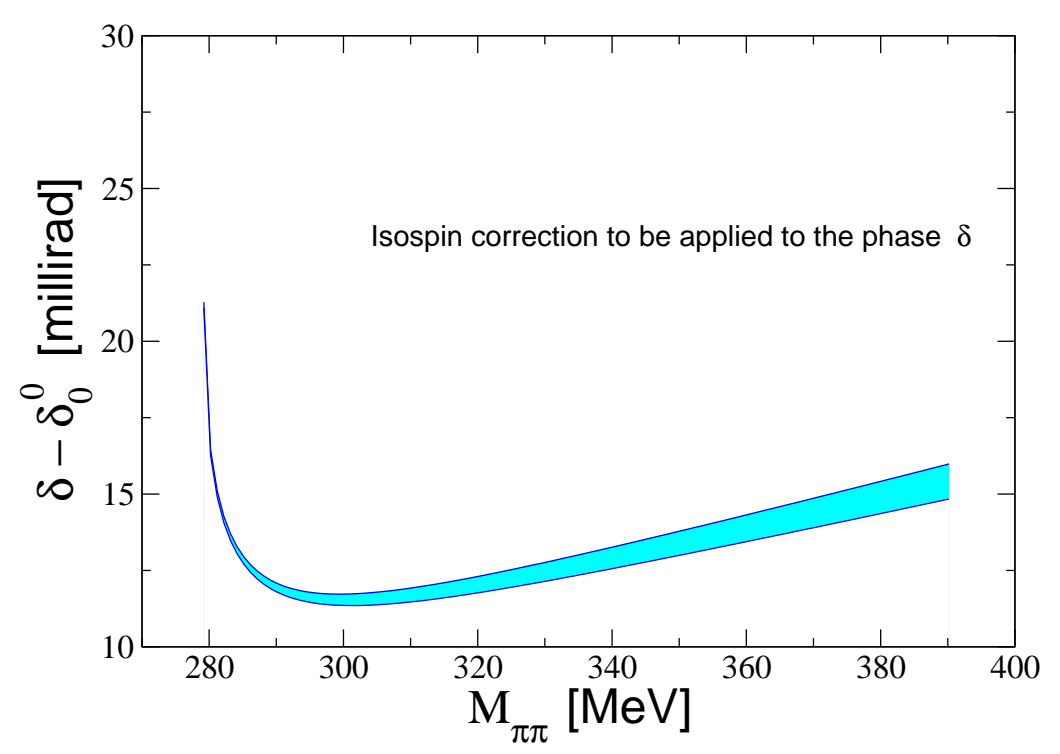

Figure 3: The isospin breaking correction that must be subtracted from the phase $\delta$ measured in $K_{e 4}$ decays. The width of the band reflects the uncertainty in the ratio $R$.

neutral and charged pions interchanged. Second, the difference $\delta-\delta_{0}^{0}$ is positive for $s_{\pi}$ above the threshold, and even increases at large $s_{\pi}$,

$$
\delta-\delta_{0}^{0}=\frac{3 s_{\pi}}{64 \pi F_{\pi}^{2}} \frac{1}{R}+O(1), \quad s_{\pi} / M_{\pi}^{2} \gg 1 .
$$

According to point iv) above, it is the phase $\delta$ that is measured in $K_{e 4}$ decays (up to contributions from higher orders in the chiral expansion). Therefore, before comparing the phase so determined with ChPT predictions, one has to subtract from the measured phase the (positive) difference $\delta-\delta_{0}^{0}$, because $\delta_{0}^{0}=\delta-\left(\delta-\delta_{0}^{0}\right)$. In Figure 3 we display this difference in the relevant decay region, for $R=37 \pm 5$. The width of the band reflects the uncertainty in $R$. It is seen that the isospin correction is substantial - it is well above the uncertainties quoted for the measured phase [47].

\subsection{The final result}

After subtracting isospin breaking corrections as discussed above, numerical solutions of Roy equations [56, 57] can again be used to extract scattering lengths from the measured phases. It turns out that also in the analysis of $K \rightarrow 3 \pi$ decays, isospin breaking corrections [65] are very important at the accuracy we are interested in [74]. Combining the result from $K_{e 4}$ decays and from the cusp analysis based on the work of [65], and using the ChPT constraint [58]

$$
a_{2}=-0.0444 \pm 0.0008+0.236\left(a_{0}-0.22\right)-0.61\left(a_{0}-0.22\right)^{2}-9.9\left(a_{0}-.022\right)^{3}
$$

to relate $a_{2}$ and $a_{0}$, the result is

$$
a_{0}=0.2196 \pm 0.0027_{\text {stat }} \pm 0.0021_{\text {syst }} \pm 0.0048_{\text {theo }}
$$




$$
\begin{aligned}
a_{2}= & -0.0444 \pm 0.0007_{\text {stat }} \pm 0.0005_{\text {syst }} \pm 0.0012_{\text {theo }} \\
a_{0}-a_{2} & =0.2640 \pm 0.0020_{\text {stat }} \pm 0.0017_{\text {syst }} \pm 0.0035_{\text {theo }} \quad[75]
\end{aligned}
$$

in very beautiful agreement with the prediction Eq. (5.1). An earlier publication which discusses the effect of these additional isospin breaking terms in the data analysis is Ref. [70].

The result of the E865 analysis Eq. (5.2) must also be corrected in view of these additional isospin breaking effects. Indeed, the scattering length (5.2) becomes smaller, and the consistency with $K_{e 4}$ and $K \rightarrow 3 \pi$ data marginal [47]. The highest energy point is responsible for most of the discrepancy. Once removed from the data set, full consistency is obtained [47]. As already mentioned, also the Geneva-Saclay data [38] lead to a consistent value when the new isospin breaking terms are included, although with a larger uncertainty. I refer the reader to the contributions of Brigitte Bloch-Devaux at Confinement08 [47] and at Kaon09 [75] for nice graphical summaries of the presently available information on the scattering lengths, see also Refs. [76].

In conclusion, all present experimental evidence supports the prediction Eq. (5.1) to an amazing degree of accuracy.

\section{Topics not covered here}

Several topics in $\pi \pi$ scattering were discussed at this conference, but are not covered here due to lack of space:

- Position of resonance poles on the second Riemann sheet [77], see also Ref. [78], where pole positions in the $\pi \pi \rightarrow \pi \pi$ amplitude were determined from first principles for the first time.

- Inverse amplitude method, unitarization of $\pi \pi$ amplitudes [79].

- Estimates of uncertainties in $\pi \pi$ amplitude at order $p^{6}$ in the chiral expansion [80].

- The use of once-subtracted Roy equations [81].

- Quark mass and $N_{C}$ dependence of the position and width of the resonance poles [82].

In addition, useful information on the $\pi \pi$ scattering amplitude may be obtained from lattice calculations, either directly, by evaluating scattering lengths, or indirectly, determining LECs that enter the chiral expansion of the $\pi \pi$ amplitude. For a recent review, I refer the interested reader to Refs. [76].

\section{Summary}

We have gone a long way, from Yukawa's prediction of a hypothetical particle responsible for the nuclear forces, up deep insight into the structure of QCD. We have witnessed a tremendous progress in theory and experiment in the field of $\pi \pi$ interaction. A coherent theoretical framework is available now, which describes this system in a very precise manner, in agreement with experimental information. 


\section{Acknowledgments}

It is a pleasure to thank the organizers for the invitation to give this talk, and for the perfect organization of the workshop and for the friendly atmosphere in Valencia. I am very grateful to Jorge Portolés for ordering the beautiful coffee cups - I now remember every morning this conference, and the good old days. I have had enjoyable discussions with Gilberto Colangelo, Bastian Kubis, Akaki Rusetsky, Zbigniew Was and with members of the NA48/2 collaboration on topics considered here.

\section{References}

[1] H. Yukawa, Proc. Phys.- Math. Soc. Jap. 17 (1935) 48.

[2] C. M. G. Lattes, H. Muirhead, G. P. S. Occhialini and C. F. Powell, Nature 159 (1947) 694; C. M. G. Lattes, G. P. S. Occhialini and C. F. Powell, Nature 160 (1947) 453; ibid. 160 (1947) 486.

[3] The state of the art about 50 years ago can be reconstructed e.g. from the Proceedings of the Rochester conference 1960 [4].

[4] Proceedings of the 1960 Annual International Conference on High Energy Physics at Rochester, E.C.G. Sudarshan, J. H. Tinlot and A. C. Melissinos (eds.), published by the University of Rochester, distributed by interscience publishers, Inc., N.Y., 1960.

[5] Yu. A. Batusov et al., in Ref. [4], p. 79.

[6] K. Ishida, A. Takahashi and Y. Ueda, Progr. Theor. Phys. 23 (1960) 731.

[7] B. S. Thomas and W. G. Holladay, Phys. Rev. 115 (1959) 1329.

[8] N. N. Khuri and S. B. Treiman, Phys. Rev. 119 (1960) 1115.

[9] R. F. Sawyer and K. C. Wali, Phys. Rev. 1191429.

[10] A. V. Efremov, V. A. Meshcheryakov and D. V. Shirkov, Sov. Phys. JETP 12 (1961) 766.

[11] J. Six and X. Artru, An essay of chronology of particle physics until 1965, in: Colloque international sur l'histoire de la physique des particules, 21-23 juillet 1982, Paris, France, Journal de Physique, 43 (1982) C8-465 [colloque C-8, supplement to Nr. 12].

[12] L. M. Brown and H. Rechenberg, The origin of the concept of nuclear forces, Institute of Physics Publishing, Bristol and Philadelphia, 1996, ISBN 0-7503-373-5.

[13] C. D. Anderson and S. H. Neddermeyer, Mesotron (Intermediate Particle) as a Name for the New Particles of Intermediate Mass, Nature 142 (1938) 878.

[14] E. Fermi, E. Teller and V. Weisskopf, Phys. Rev. 71 (1947) 314.

[15] E. Gardner and C. M. G. Lattes, Science 107 (1948) 270.

[16] J. Burfening, E. Gardner and C. M. G. Lattes, Phys. Rev. 75 (1949) 382.

[17] E. P. Wigner, Phys. Rev. 73 (1948) 1002.

[18] C. G. Goebel, Phys. Rev. Lett. 1 (1958) 337;

G. F. Chew and F. E. Low, Phys. Rev. 113 (1959) 1640.

[19] J. Schwinger, Ann. Phys. 2 (1957) 407;

F. Gürsey, Nuov. Cim. 16 (1960) 230;

J. Bernstein, M. Gell-Mann and L. Michel, Nuov. Cim. 16 (1960) 560;

M. Gell-Mann and M. Lévy, Nuov. Cim. 16 (1960) 705. 
[20] M. L. Goldberger and S. B. Treiman, Phys. Rev. 110 (1958) 1178.

[21] Y. Nambu, Phys. Rev. Lett. 4 (1960) 380.

[22] S. Treiman, A connection between the strong and weak interactions, in: Pions to quarks, Particle Physics in the 1950s, L. M. Brown, M. Dresden, L. Hoddeson, M. West (eds.), Cambridge University Press, Cambridge, 1989, ISBN 0-521-30984-0, p. 384.

[23] R. G. Newton, Phys. Rev. 114 (1958) 1611.

[24] L. Fonda and R. G. Newton, Phys. Rev. 119 (1960) 1394.

[25] L. I. Lapidus and Chou Kuang-chao, J. Exptl. Theoret. Phys. (U.S.S.R.) 39 (1960) 364 [Sov. Phys. JETP 39 (12) (1961) 258].

[26] P. Budini and L. Fonda, Phys. Rev. Lett. 6 (1961) 419.

[27] W. K. H. Panofsky, R. L. Aamodt and J. Hadley, Phys. Rev. 81 (1951) 565.

[28] N. Cabibbo, Phys. Rev. Lett. 93 (2004) 121801 [arXiv:hep-ph/0405001].

[29] J. R. Batley et al. [NA48/2 Collaboration], Phys. Lett. B 633 (2006) 173 [arXiv:hep-ex/0511056].

[30] J. L. Uretsky and T. R. Palfrey, Phys. Rev. 121, 1798 (1961).

[31] E. P. Shabalin, J. Exptl. Theor. Phys. (U.S.S.R.) 44 (1963) 765; [Sov. Phys. JETP 17 (1963) 517L]; N. Cabibbo and A. Maksymowicz, Phys. Rev. 137 (1965) B438 [Erratum-ibid. 168 (1968) 1926].

[32] E. L. Koller, S. Taylor, T. Huetter and P. Stamer, Phys. Rev. Lett. 9 (1962) 328.

[33] S. Weinberg, Phys. Rev. Lett. 17 (1966) 616.

[34] S. Weinberg, arXiv:hep-th/9702027.

[35] S. M. Roy, Phys. Lett. B 36 (1971) 353.

[36] D. Morgan and M. R. Pennington, in: L. Maiani, G. Pancheri and N. Paver, The Second Dafne Physics Handbook, INFN-LNF Divisione Ricerca, SIS-Ufficio Publicazione, Fracsati, 1995, p. 193.

[37] C. D. Froggatt and J. L. Petersen, Nucl. Phys. B 129 (1977) 89;

J. L. Petersen, Yellow Report CERN 77-04 (1977).

[38] L. Rosselet et al., Phys. Rev. D 15 (1977) 574.

[39] H. Lehmann, Phys. Lett. B 41 (1972) 529.

[40] G. Ecker and J. Honerkamp, Nucl. Phys. B 52 (1973) 211; ibid. B 62 (1973) 509.

[41] H. Fritzsch, M. Gell-Mann and H. Leutwyler, Phys. Lett. B 47 (1973) 365.

[42] J. L. Basdevant, C. D. Froggatt and J. L. Petersen, Nucl. Phys. B 72 (1974) 413.

[43] N. H. Fuchs, H. Sazdjian and J. Stern, Phys. Lett. B 269 (1991) 183;

J. Stern, H. Sazdjian and N. H. Fuchs, Phys. Rev. D 47 (1993) 3814 [arXiv:hep-ph/9301244];

M. Knecht, B. Moussallam, J. Stern and N. H. Fuchs, Nucl. Phys. B 471 (1996) 445 [hep-ph/9512404].

[44] M. Knecht, B. Moussallam, J. Stern and N. H. Fuchs, Nucl. Phys. B 457 (1995) 513 [arXiv:hep-ph/9507319]. 
[45] J. Gasser and A. Rusetsky, internal note, March 20, 2007;

J. Gasser, PoS KAON (2008) 033 [arXiv:0710.3048 [hep-ph]];

G. Colangelo, J. Gasser and A. Rusetsky, Eur. Phys. J. C 59 (2009) 777 [arXiv:0811.0775 [hep-ph]].

[46] S. R. Gevorkyan et al., arXiv:0704.2675 [hep-ph].

[47] B. Bloch-Devaux, PoS Confinement8 (2008) 029.

[48] S. Weinberg, Physica A 96 (1979) 327.

[49] J. Gasser and H. Leutwyler, Annals Phys. 158 (1984) 142.

[50] J. Gasser and H. Leutwyler, Nucl. Phys. B 250 (1985) 465.

[51] M. Luscher, Commun. Math. Phys. 105 (1986) 153.

[52] B. Adeva et al., CERN proposal CERN/SPSLC 95-1, 1995.

[53] B. Adeva et al. [DIRAC Collaboration], Phys. Lett. B 619 (2005) 50 [arXiv:hep-ex/0504044].

[54] J. Bijnens, G. Colangelo, G. Ecker, J. Gasser and M. E. Sainio, Nucl. Phys. B 508 (1997) 263 [Erratum-ibid. B 517 (1998) 639] [arXiv:hep-ph/9707291].

[55] U. G. Meißner, G. Müller and S. Steininger, Phys. Lett. B 406 (1997) 154 [Erratum-ibid. B 407 (1997) 454] [arXiv:hep-ph/9704377].

[56] B. Ananthanarayan, G. Colangelo, J. Gasser and H. Leutwyler, Phys. Rept. 353 (2001) 207 [arXiv:hep-ph/0005297].

[57] S. Descotes-Genon, N. H. Fuchs, L. Girlanda and J. Stern, Eur. Phys. J. C 24 (2002) 469 [arXiv:hep-ph/0112088].

[58] G. Colangelo, J. Gasser and H. Leutwyler, Phys. Lett. B 488 (2000) 261 [arXiv:hep-ph/0007112]; Nucl. Phys. B 603 (2001) 125 [arXiv:hep-ph/0103088].

[59] G. Amoros, J. Bijnens and P. Talavera, Nucl. Phys. B 585 (2000) 293 [Erratum-ibid. B 598 (2001) 665] [arXiv:hep-ph/0003258].

[60] G. Colangelo, PoS KAON (2008) 038 [arXiv:0710.3050 [hep-ph]].

[61] S. Pislak et al. [BNL-E865 Collaboration], Phys. Rev. Lett. 87 (2001) 221801 [arXiv:hep-ex/0106071];

S. Pislak et al. [BNL-E865 Collaboration], Phys. Rev. D 67 (2003) 072004 [arXiv:hep-ex/0301040].

[62] L. L. Nemenov, V. D. Ovsyannikov and E. V. Chaplygin, Nucl. Phys. A 710 (2002) 303.

[63] N. Cabibbo and G. Isidori, JHEP 0503 (2005) 021 [arXiv:hep-ph/0502130].

[64] E. Gamiz, J. Prades and I. Scimemi, Eur. Phys. J. C 50 (2007) 405 [arXiv:hep-ph/0602023];

K. Kampf, M. Knecht, J. Novotny and M. Zdrahal, Nucl. Phys. Proc. Suppl. 186 (2009) 334 [arXiv:0810.1906 [hep-ph]].

[65] G. Colangelo, J. Gasser, B. Kubis and A. Rusetsky, Phys. Lett. B 638 (2006) 187 [arXiv:hep-ph/0604084];

M. Bissegger, A. Fuhrer, J. Gasser, B. Kubis and A. Rusetsky, Phys. Lett. B 659 (2008) 576 [arXiv:0710.4456 [hep-ph]]; Nucl. Phys. B 806 (2009) 178 [arXiv:0807.0515 [hep-ph]].

[66] For a review, where many original references may be found, see J. Gasser, V. E. Lyubovitskij and A. Rusetsky, Phys. Rept. 456 (2008) 167 [arXiv:0711.3522 [hep-ph]]. 
[67] J. Gasser, V. E. Lyubovitskij, A. Rusetsky and A. Gall, Phys. Rev. D 64 (2001) 016008 [arXiv:hep-ph/0103157], and references cited there.

[68] B. Bloch-Devaux, Nucl. Phys. Proc. Suppl. 174 (2007) 91.

[69] Talks from the NA48/2 collaboration may be found at http://na48.web.cern.ch/NA48/Welcome/images/talks.html

[70] J. R. Batley et al. [NA48/2 Collaboration], Eur. Phys. J. C 54 (2008) 411.

[71] E. Barberio, B. van Eijk and Z. Was, Comput. Phys. Commun. 66 (1991) 115;

E. Barberio and Z. Was, Comput. Phys. Commun. 79 (1994) 291;

G. Nanava and Z. Was, Eur. Phys. J. C 51 (2007) 569 [arXiv:hep-ph/0607019].

[72] B. Bloch-Devaux and Z. Was, private communications.

[73] V. Cuplov and A. Nehme, arXiv:hep-ph/0311274;

A. Nehme, Phys. Rev. D 69 (2004) 094012 [arXiv:hep-ph/0402007]; Phys. Rev. D 70 (2004) 094025 [arXiv:hep-ph/0406209]; Eur. Phys. J. C 40 (2005) 367 [arXiv:hep-ph/0408104].

[74] D. Madigozhin, talk given at KAON09, to appear in the proceedings.

[75] B. Bloch-Devaux, talk given at KAON09, to appear in the proceedings.

[76] H. Leutwyler, Int. J. Mod. Phys. A 23 (2008) 3187;

H. Leutwyler, PoS Confinement8 (2008) 068 [arXiv:0812.4165 [hep-ph]];

G. Colangelo, talk given at PANIC08, to appear in the proceedings.

[77] J. R. Peláez, talk given at this conference;

R. García-Martín, R. Kamiński and J. R. Peláez, Int. J. Mod. Phys. A 24 (2009) 590 [arXiv:0810.1134 [hep-ph]].

[78] I. Caprini, G. Colangelo and H. Leutwyler, Phys. Rev. Lett. 96 (2006) 132001

[arXiv:hep-ph/0512364].

[79] H. Q. Zheng, talk given at this conference; arXiv:0905.0528 [hep-ph].

[80] J. J. Sanz-Cillero, talk given at this conference;

Z. H. Guo and J. J. Sanz-Cillero, Phys. Rev. D 79 (2009) 096006 [arXiv:0903.0782 [hep-ph]].

[81] R. García-Martín, talk given at this conference;

R. Kamiński, R. García-Martín, J. R. Peláez and F. J. Ynduráin, arXiv:0905.2139 [hep-ph];

R. Kamiński, R. García-Martín, P. Grynkiewicz and J. R. Peláez, Nucl. Phys. Proc. Suppl. 186 (2009) 318 [arXiv:0811.4510 [hep-ph]];

R. Kamiński, R. García-Martín, P. Grynkiewicz, J. R. Peláez and F. J. Ynduráin, Int. J. Mod. Phys. A 24 (2009) 402 [arXiv:0809.4766 [hep-ph]].

[82] G. Rios Márquez, talk given at this conference;

G. Rios Márquez, A. Gómez Nicola, C. Hanhart and J. R. Peláez Sagredo, arXiv:0905.3489 [hep-ph]. 